\title{
PENETRATION OF HYDROPHILIC SULFORHODAMINE B ACROSS THE PORCINE CORNEA EX-VIVO
}

\author{
WANACHAT CHAIYASAN ${ }^{*}$, SANGLY P. SRINIVAS ${ }^{b}$, PATTRAVEE NIAMPREMc, WAREE TIYABOONCHAI ${ }^{c, d, e}$
}

aDepartment of Optometry, Faculty of Allied Health Sciences, Naresuan University, Phitsanulok, Thailand, bSchool of Optometry, Indiana University, Bloomington, IN, United States, cDepartment of Pharmaceutical Technology, Faculty of Pharmaceutical Sciences, Naresuan University, Phitsanulok, Thailand, dThe Center of Excellence for Innovation in Chemistry (PERCH-CIC), Commission on High-Education, Ministry of Education, Thailand, The Center of Excellence in Medical Biotechnology, Naresuan University, Phitsanulok, Thailand Email: wanachatch@nu.ac.th

Received: 14 Jul 2018, Revised and Accepted: 04 Sep 2018

ABSTRACT

Objective: Sulforhodamine B (SRB) is a hydrophilic tracer whose fluorescence is unaffected by pH unlike that of carboxyfluorescein. Therefore, SRB may serve as a better tracer when there are significant changes in $\mathrm{pH}$. Thus, in this study, the suitability of SRB to assess the barrier properties of the cellular layers of the cornea was examined using a custom-built confocal scanning micro-fluorometer (CSMF).

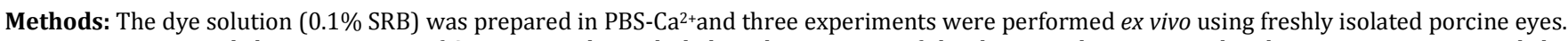
First, we investigated the penetration of SRB across the endothelium by injection of the dye into the anterior chamber. Next, we measured the penetration of SRB across the epithelium after exposing the ocular surface to the dye. Finally, we examined the penetration after exposure to the dye with detergent (Tween 20) and exposure to the dye concomitant with microneedle injuries. The dye concentration profiles across the cornea were measured using CSMF.

Results: SRB penetrated the corneal endothelium readily into the stroma following injection into the anterior chamber in a time-dependent manner. Despite accumulation in the stroma, SRB did not partition into the epithelium. In agreement with these findings, the dye did not cross the epithelium after topical administration. Co-administration with Tween 20 and injury to the epithelium with microneedles, however, led to penetration of the dye into the stroma.

Conclusions: SRB is a hydrophilic dye that can be used as an alternative fluorescent tracer to assess the barrier function of the cellular layers of the cornea.

Keywords: Sulforhodamine B, Cornea, Endothelium, Epithelium, Confocal scanning microfluorometry

(C) 2018 The Authors. Published by Innovare Academic Sciences Pvt Ltd. This is an open-access article under the CC BY license (http://creativecommons.org/licenses/by/4.0/) DOI: http://dx.doi.org/10.22159/ijap.2018v10i6.28505

\section{INTRODUCTION}

The cornea is the main refracting element of the eye. Therefore, its transparency is critical for acute vision. The transparency of the cornea is dependent on the stromal deturgescence (i.e., a state of dehydration of the stroma). The stromal deturgescence is maintained by the barrier and fluid pump functions of the corneal endothelium [1]. The resistance to a persistent fluid leak that occurs from the anterior chamber into the stroma is the barrier function of the endothelium. The mechanism that counterbalances the residual leak is the fluid pump activity. Thus, both the barrier and pump functions are obligatory for the stromal deturgescence. The pump function entails water movement by the transcellular route, and it is driven by local osmotic gradients induced by vectorial solute transport mechanisms of the endothelium. The barrier function, on the other hand, refers to passive fluid flux via the paracellular route. Thus, the barrier function is determined by the integrity of the tight junctions of the endothelium. Since the electrical methods are not suitable in vivo, the integrity of the tight endothelial junctions is frequently measured by permeability to hydrophilic solutes [2-5]. Previously, hydrophilic dyes such as sodium fluorescein (MW = 376; $\log \mathrm{P}=-1.52$ ) and its derivative carboxyfluorescein ( $\mathrm{MW}=378$ $\mathrm{g} / \mathrm{mol}, \log \mathrm{P}=-1.5$ ) have been employed to characterize the barrier function of the corneal endothelium in a clinical setting using an ocular fluorometer $[3,6,7]$.

Both fluorescein and carboxyfluorescein exhibit many useful characteristics as non-toxic polar solutes. They possess excitation and emission spectra in the visible range and exhibit high quantum yield. Fluorescein is the dye of choice for human studies as it is approved for use in clinical practice [8]. However, in animal studies, carboxyfluorescein is the dye of choice since it is relatively more hydrophilic than fluorescein. Because of higher hydrophilicity, the permeability of the endothelium to carboxyfluorescein would be a better measure of the integrity of the tight junctions [9]. However, the fluorescence spectra of carboxyfluorescein are sensitive to $\mathrm{pH}$ [10]. Thus, measurements of its fluorescence and hence the calculated permeability are confounded by changes in stromal $\mathrm{pH}$.

Sulforhodamine B (SRB) is a hydrophilic dye (MW $=581$; $\log \mathrm{P}=-$ 2.02; water solubility $=10 \mathrm{~g} / 100 \mathrm{ml}$ ) [11] with absorption and emission peaks at 565 and $585 \mathrm{~nm}$, respectively. More importantly, the fluorescence of SRB is unaffected by $\mathrm{pH}$ in the physiological range $[12,13]$. Therefore, SRB is a better solute for permeability measurements compared to fluorescein or carboxyfluorescein. Previously, the penetration of SRB into the rabbit corneal epithelium has been reported [14]. Although SRB penetrated into the stroma, the dye was not found to stain the epithelial cells as observed by fluorescence microscopy [14]. In another study, SRB was found to penetrate into mouse corneas, which were exposed to toxic substances and had caused damage to the epithelium [15]. However, the penetration of SRB across the porcine cornea (which is similar to the human cornea $[16,17]$ has not been reported. Therefore, the objective of this study was to explore SRB as an alternative fluorescent dye to assess the barrier integrity of the epithelial and endothelial layers of the porcine cornea. We have measured the penetration of SRB with porcine cornea ex vivo using a custom-made confocal scanning microfluorometer (CSMF) [18-21]. Our results indicate that SRB can be a superior dye for the assessment of permeability of the cellular barriers of the cornea.

\section{MATERIALS AND METHODS}

\section{Materials}

SRB and penicillin/streptomycin were obtained from Sigma Chemical Co (St. Louis, Mo, U.S.A). All other chemicals and solvents were of analytical grade. 


\section{Preparation of porcine eyeballs}

Porcine eyeballs were collected from a local slaughterhouse and transported to the laboratory within $2 \mathrm{~h}$ immersed in ice-cold phosphate buffered saline (PBS with $\mathrm{Ca}^{2+} ; \mathrm{pH} 7.4$ ) containing 200 $\mu \mathrm{g} / \mathrm{ml}$ of penicillin/streptomycin. Eyeballs, without any injury to the epithelium, were placed in a moisture chamber at room temperature prior to use in the permeability assay.

\section{Confocal scanning microfluorometer (CSMF)}

The CSMF, which has been described previously [20, 21], was employed to assess the transcorneal penetration of SRB. The instrument is equipped with a water-immersion objective (Zeiss 40x; $0.75 \mathrm{NA}$ and $\mathrm{wd}=1.2 \mathrm{~mm}$ ) and measures transcorneal fluorescence profiles with a depth resolution of $7 \mu \mathrm{m}$. All depth scans were performed at $10 \mu \mathrm{m} / \mathrm{s}$. The excitation was obtained by a white light emitting diode (LED). The output of the LED (10W; XML series; Cree Inc) was modulated at $10 \mathrm{kHz}$ and filtered through an interference filter $(565+10 \mathrm{~nm})$. The output was coupled to the circular end of a fiber optic bundle. At the other end of the bundle, the fibers were placed in a v-groove to form an excitation slit. The SRB fluorescence $(>585 \mathrm{~nm})$ and scattered light were collected through a parfocal slit positioned in the eyepiece. A photomultiplier tube (R928HA Hamamatsu) and a photodiode (S1226-5BQ; Hamamatsu), coupled to two independent lock-in amplifiers, detected the fluorescence and scattered light, respectively. As shown in fig. 1, all measurements were performed with eyeballs positioned underneath the objective on a motorized XYZ translation stage (Newport Inc., USA). The fluorescence/scatter scans were performed under computer control using custom-made Lab VIEW software (National Instruments).

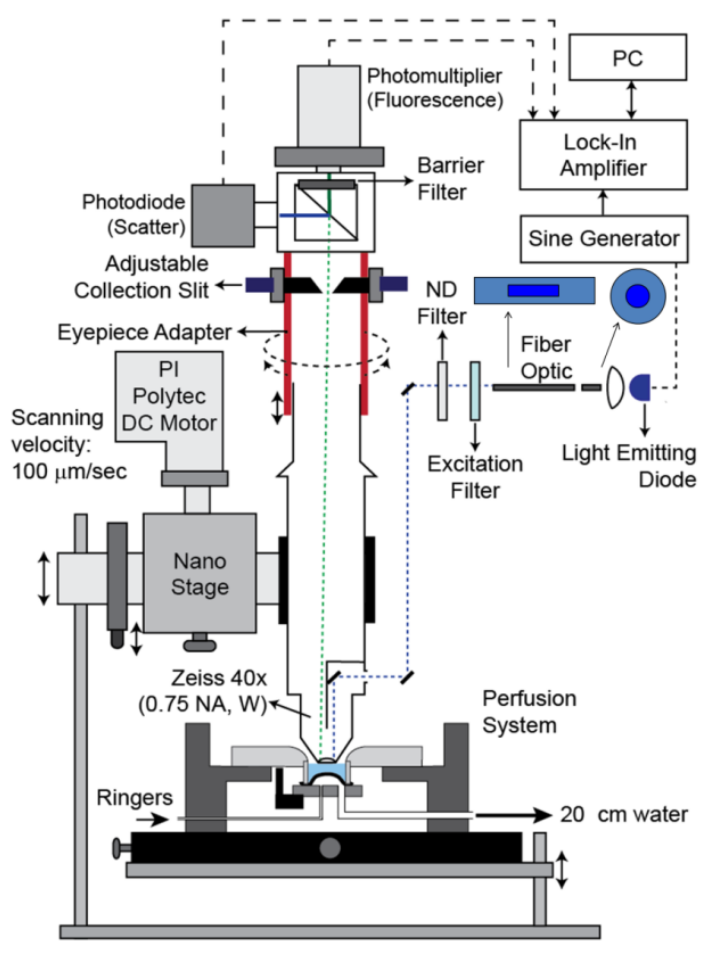

Fig. 1: Confocal scanning micro fluorometer employed for the measurements of transcorneal penetration of SRB $[20,21]$

\section{Penetration of SRB}

Three groups of experiments were performed to characterize the transient penetration of SRB across the (a) endothelium, (b) cornea with and without epithelium, and (c) injured epithelium. For the latter experiments, the epithelium was injured with microneedles or by exposure to detergent. For all experiments, $0.1 \%$ SRB solution was prepared in PBS with $\mathrm{Ca}^{2+}(\mathrm{pH}$ 7.4). Each group of experiments was repeated in at least six eyes.
For characterization of transient penetration of SRB across the endothelium, SRB solution $(40 \mu \mathrm{l})$ was injected slowly using a 27 gauge needle into the anterior chamber. The injection was made $\sim 2$ $\mathrm{mm}$ posterior to the limbus. The needle tip did not touch the endothelium during the injection. The fluorescence and scatter signals were recorded subsequently using the CSMF for up to $100 \mathrm{~min}$.

For characterization of the transient penetration of SRB across the cornea with and without epithelium, $100 \mu \mathrm{l}$ of SRB solution was placed at the center of the cornea for $0.5,1,4$, and $12 \mathrm{~h}$ in a dark, moist chamber at room temperature. For some corneas, the epithelium was debrided using a sterile stainless-steel blade before exposure to the SRB solution. After each incubation period, the eyeballs were washed three times with PBS (containingCa ${ }^{2+} ; \mathrm{pH}$ 7.4) and subsequently the fluorescence and scatter profiles were recorded using the CSMF as before.

For characterization of the transient penetration of SRB into the stroma of corneas with compromised epithelium, we employed Tween 20 to break down the tight junctions and independently, using microneedles. Thus, in some corneas, the epithelium was exposed to $100 \mu \mathrm{l}$ of SRB solution containing $0.5 \%$ Tween 20 (dissolving in PBS with $\mathrm{Ca} 2+\mathrm{pH} 7.4$ ) for 6-12 $\mathrm{h}$. In other corneas, a 27-gauge needle was used to make several microscopic injuries to the epithelium before exposure to $100 \mu \mathrm{l}$ of the SRB solution for $6 \mathrm{~h}$. After incubation, the eyeballs were washed three times with PBS as above and then fluorescence and scatter profiles were recorded using the CSMF.

\section{RESULTS}

\section{Penetration of SRB across the endothelium}

The transcorneal profiles of SRB fluorescence from a typical experiment at various intervals between 30-100 min after injection of SRB into the anterior chamber are shown in fig. 2. The SRB fluorescence across the stroma increased in a time-dependent manner indicating significant permeability of the dye across the corneal endothelium and consequent accumulation in the stroma. Despite the accumulation of the dye in the stroma to very high levels, it did not subsequently penetrate into the epithelium. As shown in fig. 2, a typical corneal scatter versus depth was taken at $10 \mathrm{~min}$ after injecting the dye. The peak heights between the scatter from the epithelium and endothelium can be taken as the thickness of the stroma, which was found to be about $650 \mu \mathrm{m}(\mathrm{n}=6)$.

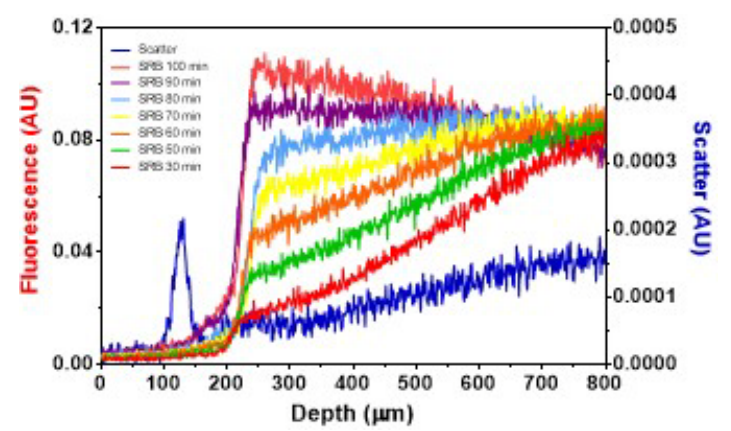

Fig. 2: Penetration of SRB across the corneal endothelium after injection of the dye into the anterior chamber of with porcine eyes ex vivo. The transcorneal fluorescence and scatter profiles were obtained $100 \mathrm{~min}$ after injection of SRB $(0.1 \%)$. Results

shown are similar to 6 other independent experiments

\section{Penetration of SRB across the epithelium}

The transcorneal profiles of SRB fluorescence with and without epithelium are shown in fig. 3 and fig. 4, respectively. As noted in fig. 3 , there is no increase in SRB fluorescence within the corneal stroma even after $12 \mathrm{~h}$ of exposure to SRB at the ocular surface. This indicates that SRB does not penetrate the epithelium. The apparent peak in SRB fluorescence at the epithelium in fig. 3 is due to adherent dye as the corneal surface could not be washed rigorously to prevent damage to the epithelium. In contrast to the findings in 
fig. 3, in the case of the cornea without the epithelium, we found SRB to rapidly partition into the stroma within $30 \mathrm{~min}$ (fig. 4). Thus, the data in fig. 3 and fig. 4 , taken together, indicate that the epithelium is a barrier to the penetration of the hydrophilic SRB.

The observations in fig. 3 and 4 have been further confirmed with models of injury to the corneal epithelium. As the first model, we damaged the tight junctions of the epithelium by exposure to $0.5 \%$ Tween 20, a nonionic detergent. As shown in fig. 5, the transcorneal fluorescence of SRB increased significantly in the presence of the detergent. In the second model, we caused mechanical injury to the epithelium using a microneedle. Similar to Tween 20, penetration of SRB also increased after the microneedle injury to the epithelium as shown in fig. 6.

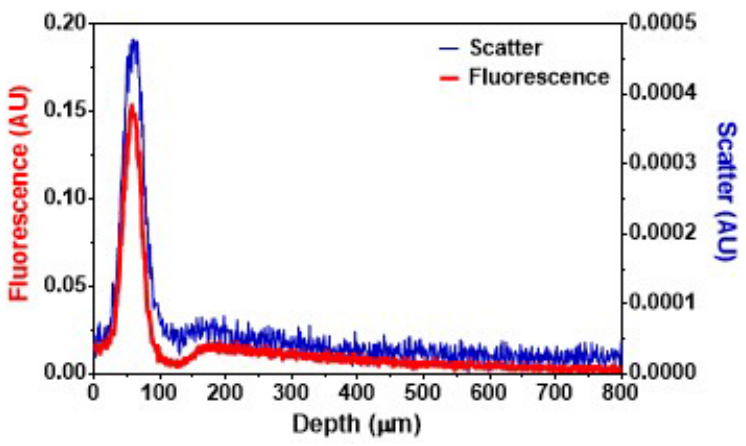

Fig. 3: Penetration of topical SRB with intact epithelium, the transcorneal fluorescence and scatter profiles $12 \mathrm{~h}$ after application of SRB (0.1\%) to the epithelial surface. Results shown are similar to findings in 6 other independent experiments

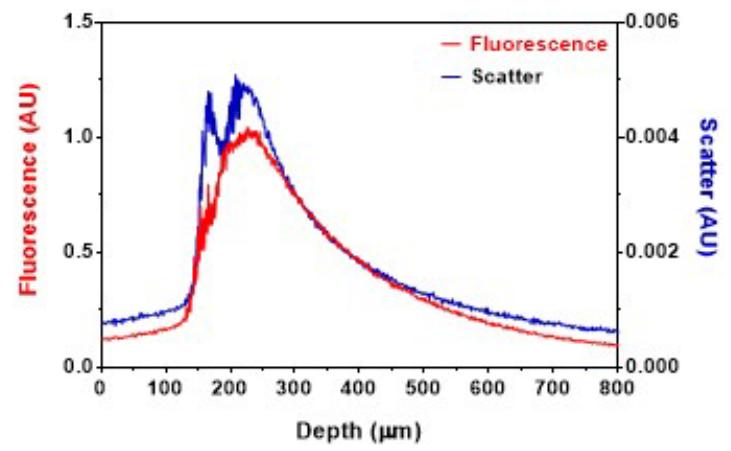

Fig. 4: Penetration of topical SRB after removal of the corneal epithelium, the transcorneal fluorescence and scatter profiles 30 min after application of SRB on bare stroma. Results shown are similar to findings in 6 other independent experiments

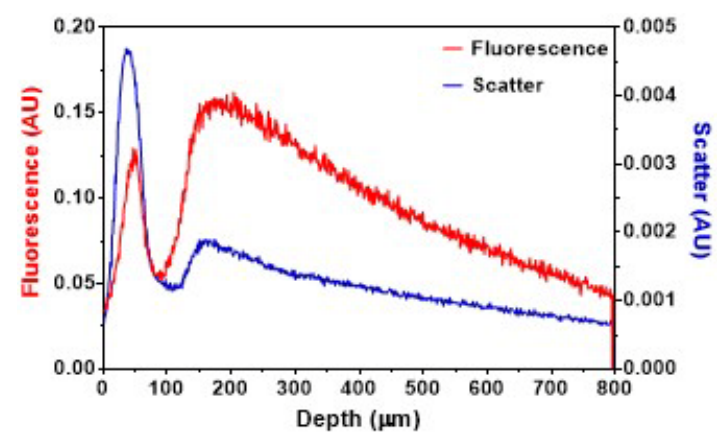

Fig. 5: Penetration of SRB after disruption of the tight junctions of the epithelium, the transcorneal fluorescence and scatter profiles $12 \mathrm{~h}$ after application of SRB with Tween $20(0.5 \%)$ at the epithelial surface, results shown are similar to findings in 6 other independent experiments

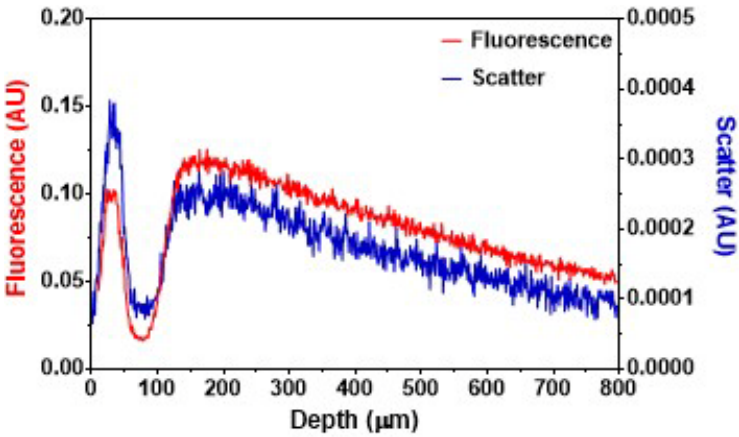

Fig. 6: Penetration of SRB after disruption of corneal epithelium using needles, the transcorneal fluorescence and scatter profiles $12 \mathrm{~h}$. Results shown are similar to findings in 6 other independent experiments

\section{DISCUSSION}

Most ophthalmic drugs are administered as drops on the ocular surface [22]. Hence, the studies on drug penetration across the cornea are vital to the rational design of ophthalmic drugs and their delivery strategies [23-25]. The corneal epithelium consists of 5-7 layers of cells. The superficial two layers are thought to contain a significant number of tight junctional strands, and hence form a barrier for hydrophilic solutes. On the other hand, the monolayer of corneal endothelium at the posterior surface of the cornea is very leaky. The stroma, which contains $80 \%$ water in a collagenous matrix, would be opposed to the accumulation of hydrophobic solutes but may form a depot for hydrophilic drugs. In this context, the permeability of SRB, a pH-insensitive hydrophilic molecule, into the porcine cornea has been evaluated using the custom-made CSMF. The data not only highlight the cellular barriers for transcorneal penetration of drugs but also predictive of the integrity of the cellular layers.

Our experiments have been performed with the freshly isolated porcine cornea, which forms an excellent model, similar to rabbits, for studies on the transcorneal penetration of drugs and solutes [7, 26]. The porcine cornea is structurally similar to the human cornea $[17,27]$, although thicker than the human cornea [28]. The thickness of the porcine cornea is reported to be $\sim 1010 \mu \mathrm{m}$ [29]. In our experiments, the thickness, measured as the distance between peaks corresponding to the epithelium and endothelium from the scatter plots, was $\sim 900 \mu \mathrm{m}$ (ranged from 800 to $1000 \mu \mathrm{m} ; \mathrm{n}=12$ ).

The penetrations of hydrophilic solutes into the corneal stroma are dependent on the integrity of the tight junctions of the corneal epithelium and endothelium. Thus, to assess the status of tight junctions, which is affected by inflammation or cell death, the permeability to hydrophilic dyes has been measured. For these investigations, an ideal dye would have $\mathrm{pH}$-insensitive fluorescence properties along with non-cytotoxicity to the corneal epithelium or endothelium. SRB is similar to carboxyfluorescein, which has long been used as a hydrophilic tracer for characterizing paracellular flux exclusively [30]. The data in fig. 3 and fig. 5 demonstrate that SRB penetrates the cornea after topical instillation exclusively through the paracellular pathway. As evident by data in fig. 3 and 4 together, SRB does not penetrate the corneal epithelium. This is in contrast to the Rhodamine B, which is relatively lipophilic [31]. In agreement with our findings in fig. 3 and 4, SRB partitioned very well into the stroma once the epithelium was removed (similar to sodium fluorescein that stains the cornea devoid of the epithelium [32]) (fig. 4) or when the integrity of the epithelium was disrupted by Tween 20 (fig. 5). The latter observation is reminiscent of the finding that Tween $20(1 \%)$ induced an increase in the penetration of fluorescein into the human eyes [33]. The breakdown of the tight junctions at the concentration of Tween 20 employed in this study has also been reported previously for Caco-2 cells [34]. The hydrophilic characteristics of the SRB are further demonstrated by the fact that the dye does not penetrate the epithelium, even after significant accumulation in the stroma (fig. 1). Finally, as expected, we found 
that the microneedle injury enhanced the permeability of SRB across the epithelium (fig. 6).

Collectively, our observations indicate that SRB can be used as an alternative hydrophilic tracer to assess the barrier functions of the corneal epithelium and endothelium. The characteristics of SRB penetration from the anterior chamber are similar to that of carboxyfluorescein as reported previously with the CSMF [1].

\section{CONCLUSION}

The findings of our study indicate that SRB could be employed as an alternative hydrophilic tracer to assess the barrier functions of the corneal epithelium and endothelium ex vivo. SRB is insensitive to $\mathrm{pH}$, and hence, could be more advantageous as a tracer for assessment of paracellular permeability.

\section{ACKNOWLEDGMENT}

The authors gratefully acknowledge financial support by the Naresuan University Research Fund, Thailand (Grant number R2560C038).

\section{AUTHORS CONTRIBUTIONS}

All the authors have contributed equally

\section{CONFLICTS OF INTERESTS}

The authors declare that there is no conflict of interest

\section{REFERENCES}

1. Srinivas SP. Dynamic regulation of barrier integrity of the corneal endothelium. Optom Vis Sci 2010;87:239-54

2. Srinivas SP. Cell signaling in regulation of the barrier integrity of the corneal endothelium. Exp Eye Res 2012;95:8-15.

3. Srinivas SP, Goyal A, Talele DP, Mahadik S, Sudhir RR, Murthy $\mathrm{PP}$, et al. Corneal epithelial permeability to fluorescein in humans by a multi-drop method. PLoS One 2018;13:e0198831.

4. Nagataki S, Brubaker RF, Grotte DA. The diffusion of fluorescein in the stroma of rabbit cornea. Exp Eye Res 1983;36:765-71.

5. Srinivasan B, Kolli AR, Esch MB, Abaci HE, Shuler ML, Hickman JJ. Teer measurement techniques for in vitro barrier model systems. J Lab Autom 2015;20:107-26.

6. Araie M, Maurice D. The rate of diffusion of fluorophores through the corneal epithelium and stroma. Exp Eye Res 1987;44:73-87.

7. Gupta C, Chauhan A, Srinivas SP. Penetration of fluorescein across the rabbit cornea from the endothelial surface. Pharm Res 2012;29:3325-34.

8. Robertson TA, Bunel F, Roberts MS. Fluorescein derivatives in intravital fluorescence imaging. Cells 2013;2:591-606.

9. Araie M. Carboxyfluorescein. A dye for evaluating the corneal endothelial barrier function in vivo. Exp Eye Res 1986;42:141-50.

10. Mordon S, Devoisselle JM, Maunoury V. In vivo ph measurement and imaging of tumor tissue using a ph-sensitive fluorescent probe (5,6-carboxyfluorescein): Instrumental and experimental studies. Photochem Photobiol 1994;60:274-9.

11. Amato DV, Lee H, Werner JG, Weitz DA, Patton DL. Functional microcapsules via thiol-ene photopolymerization in dropletbased microfluidics. ACS Appl Mater Interfaces 2017;9:3288-93.

12. Behrendorff N, Shukla A, Schwiening C, Thorn P. Local dynamic changes in confined extracellular environments within organs. Clin Exp Pharmacol Physiol 2009;36:1010-5.

13. Schulz A, Hornig S, Liebert T, Birckner E, Heinze T, Mohr GJ. Evaluation of fluorescent polysaccharide nanoparticles for phsensing. Org Biomol Chem 2009;7:1884-9.
14. Chodosh J, Dix RD, Howell RC, Stroop WG, Tseng SC. Staining characteristics and antiviral activity of sulforhodamine $\mathrm{b}$ and lissamine green b. Invest Ophth Vis Sci 1994;35:1046-58.

15. Maurice D, Singh T. A permeability test for acute corneal toxicity. Toxicol Lett 1986;31:125-30.

16. Hara $\mathrm{H}$, Cooper DKC. Xenotransplantation-the future of corneal transplantation? Cornea 2011;30:371-8.

17. Elsheikh A, Alhasso D, Rama P. Biomechanical properties of human and porcine corneas. Exp Eye Res 2008;86:783-90.

18. Wang Y, Gabe K, Kompella UB, Barbieri B, Srinivas SP. Confocal scanning microfluorometer for transcorneal fluorescence lifetime measurements. Invest Ophth Vis Sci 2012;53:3093.

19. Srinivas SP, Chaiyasan W, Niamprem P, Keefer K, Tiyaboonchai W, Kompella UB. Penetration of polar sulforhodamine $b$ into the cornea. Invest Ophth Vis Sci 2015;56:4138.

20. Maurice DM, Srinivas SP. Fluorometric measurement of light absorption by the rabbit cornea. Exp Eye Res 1994;58:409-13.

21. Srinivas SP, Maurice DM. A microfluorometer for measuring diffusion of fluorophores across the cornea. IEEE Trans Biomed Eng 1992;39:1283-91.

22. Gaudana R, Ananthula HK, Parenky A, Mitra AK. Ocular drug delivery. AAPS J 2010;12:348-60.

23. Niamprem P, Srinivas SP, Tiyaboonchai W. Development and characterization of indomethacin-loaded mucoadhesive nanostructured lipid carriers for topical ocular delivery. Int J Appl Pharm 2018;10:91-6.

24. Chaiyasan W, Srinivas SP, Tiyaboonchai W. Development and characterization of topical ophthalmic formulations containing lutein-loaded mucoadhesive nanoparticles. Int J Pharm Pharm Sci 2016;8:261-6.

25. Morsi N, Ghorab D, Refai H, Teba H. Preparation and evaluation of alginate/chitosan nanodispersions for ocular delivery. Int J Pharm Pharm Sci 2015;7:234-40.

26. Chaiyasan W, Praputbut S, Kompella UB, Srinivas SP, Tiyaboonchai W. Penetration of mucoadhesive chitosandextran sulfate nanoparticles into the porcine cornea. Colloids Surf B 2017; 149:288-96.

27. Marquez-Curtis LA, McGann LE, Elliott JAW. Expansion and cryopreservation of porcine and human corneal endothelial cells. Cryobiology 2017;77:1-13.

28. Sanchez I, Martin R, Ussa F, Fernandez Bueno I. The parameters of the porcine eyeball. Graefes Arch Clin Exp Ophthalmol 2011;249:475-82.

29. Jay L, Brocas A, Singh K, Kieffer JC, Brunette I, Ozaki T. Determination of porcine corneal layers with a high spatial resolution by simultaneous second and third harmonic generation microscopy. Opt Express 2008;16:16284-93.

30. Stiemke MM, McCartney MD, Cantu-Crouch D, Edelhauser HF. Maturation of the corneal endothelial tight junction. Invest Ophth Vis Sci 1991;32:2757-65.

31. Gupta C, Chauhan A, Mutharasan R, Srinivas SP. Measurement and modeling of diffusion kinetics of a lipophilic molecule across rabbit cornea. Pharm Res 2010;27:699-711.

32. Annear MJ, Petersen-Jones SM. Surgery of the ocular surface. In: Auer JA, Stick JA. editors. Chapter 57. Equine surgery (fourth edition). Saint Louis: W. B. Saunders; 2012. p. 770-92.

33. Marsh RJ, Maurice DM. The influence of non-ionic detergents and other surfactants on human corneal permeability. Exp Eye Res 1971;11:43-8.

34. Ujhelyi Z, Fenyvesi F, Varadi J, Feher P, Kiss T, Veszelka S, et al. Evaluation of cytotoxicity of surfactants used in self-micro emulsifying drug delivery systems and their effects on paracellular transport in caco-2 cell monolayer. Eur J Pharm Sci 2012;47:564-73. 